\title{
Thinking of Muscle Strength Training Method
}

\author{
Zhongfeng Sun ${ }^{1}$ \\ ${ }^{1}$ Department of Physical Education, Qingdao University of Science \& Technology, Laoshan District, Qingdao, \\ Shandong, China \\ Correspondence: Zhongfeng Sun, Department of Physical Education, Qingdao University of Science \& \\ Technology, Laoshan District, Qingdao 266061, Shandong, China. E-mail: sunzhongfeng1976@163.com
}

Received: April 19, 2015 Accepted: April 28, 2015 Online Published: April 30, 2015

doi:10.5539/ass.v11n13p12 URL: http://dx.doi.org/10.5539/ass.v11n13p12

\begin{abstract}
When we analyse the principle of strength training, or the design of the various training methods, it will be found that reasonable and suitable training design, principles and technology should be designed for most people, if they want to increase their strength. In this paper, we discuss the basic principles and the training methods which should be mastered, taken as a basis for strength training plan formulation.
\end{abstract}

Keywords: strength training, principles of strength training, strength training methods

\section{The General Principle of Strength Training}

There are four common principles should be executed unremittingly, no matter which kind of training methods you use, isometric training, isotonic training or isodynamic training.

1) Training plan should be made for the muscles which need to be strengthened, and the load weight should be increased gradually in the process of training. Firstly, we must find out which muscle needs to be reinforced. One simple method is that you can press or touch the muscle on both ends of the joint, when practitioners do actions. It is the muscle completing this action when you feel it becomes nervous and hard in the action. If you want to strengthen the muscle, large resistance method can be used to train it.

2) You should make the training as enjoyably as you can. One of the main aspects of a high quality training plan can make the trainers to maintain a high level of enthusiasm. On the contrary, a bad training plan will make the trainers annoyed and uninterested. No matter how big and profound your plan is in the aspect of theory, it is equivalent to failure if the trainers don't want to stick to.

3) We should train big muscles firstly before training small muscles. General speaking, the small muscles fatigue more easily than the larger muscle groups. When the muscles fatigue, the training effect will be worse and worse, and there will be not any effect finally. It is very important to train the larger muscle groups related to the action firstly. For example, weightlifters should practice big muscles in the legs firstly, and then practice stretch elbow muscle groups.

4) Enough time is needed for muscles to recover from fatigue between the training cycles and between each group of practices. This principle is related to the former principle. If the muscles are in a state of fatigue and pain, or have not yet recovered from fatigue after the previous practice, it is difficult to get a good training effect in this case. Therefore, when we arrange strength training, we should pay attention to avoid the fault making the load act on the same muscles in some continuous practice.

\section{The General Principles of Isometric Training}

After system research, we can find that it is quick and easy to improve muscle strength with isometric training. That is to say, people can quickly improve the level of power with very little practice. But shown in the subsequent experience and further research, the isometric training is also flawed. We will discuss the defects later, now let's talk about the basic principle of isometric training.

The effect of isometric training largely depends on the chosen angle of activity. For example, the elbow flexion overload movement may have the best effect when the angle is $90^{\circ}$. We should assign the angle order if a certain angle is weak, practice at an angle firstly, and then practice at another angle in order to make the isometric training to improve muscle strength at any angle. 
Every contraction should be maximum voluntary contraction. Some material shows that the training effect of $50 \%$ of the maximum force is better; however, it doesn't work in fact, because it is impossible to measure the greatest power of each muscle firstly before each training, and then give $50 \%$ load. Generally speaking, it can develop muscle strength for trainers to use the greatest endeavor with a gradual increase in load.

The biggest contraction often can be hold for 10 seconds, generally the muscle do not fatigue within 10 seconds. But some research also proved that the training also won't have obvious effect if the duration of the biggest contraction has more two to five seconds. Sore muscles is connected with muscle fatigue, in this case there would be no better reason to extend the biggest muscle contraction times.

Isometric contraction should be conducted repeatedly 1-5 times in every training session, practice should be taken every day unless the abnormal pain of muscle occurs. 2 to 3 minutes of recovery time should be given after the biggest force in the muscle is taken every time, during the recovery time we can train other muscles. Because the time of isometric training is often short, it consumes less energy than isotonic training and isodynamic training, and the muscle sore is not easy to happen, so the rest time between isometric training can be shorter. Study also found that the isometric training effect of three to four times a week is just like the effect of every day training, it can also increase strength finally only with slow progress.

It will be able to maintain the growth strength if we take one normal isometric training every week, it is often more difficult for all kinds of physical training to increase force than hold the strength, the strength training is no exception. The suggestion of training once a week is more cautious, because some authority figures in their research found that it also can keep the growth force even if we practice once every two weeks with 2 seconds of the biggest contraction at a time. Of course, it is secure for us to adopt a more cautious approach. Certainly, the growth force will return to the beginning of the original level usually within 1 to 2 months, if we don't practice again after the strength has been growth, $40 \%$ of the growth strength can be lost, but it will require a year or longer time if you want to grow this $40 \%$ of the strength.

It is necessary for the isometric training plan to have fun, not be boring. We should pay attention to practice big muscles firstly, and then practice the small muscles, because small muscle fatigue is easier to happen.

\section{The General Principles of Isotonic Training}

In addition to the normal muscle activities every day, it can increase the strength of the muscle to plus some excess load.

3-4 group practice with maximum weight should be taken after each session of practice; the maximum weight is lifted 1-6 times in each group. The standard of overload is marked as the most repeat times (RM). A RM is the maximum weight raised one time; Two RM is the maximum weight two times, and so on. It is obvious that the more the RM is, the heavier the weight can be lifted. Some research proves that the practice method using less times to close to the maximum weight can increase the maximum strength. Many famous experts believe that the most satisfying training method is to lift 1-6 RM, practice three to four groups, and take this as a principle of training. After the training is more than $6 \mathrm{RM}$, the training effect of muscle strength will be gradually reduced, but the effect for developing anaerobic endurance of muscle will be better. The effect of centrifugal contraction is poorer than the centripetal contraction in isotonic training, though the practice has power using the centrifugal contraction method, the strength is offset by the defect that using centrifugal contraction is easy to make sore muscles. According to the contrast test, it must be clear how much weight is right within certain repetitions. Generally, it will gradually increase muscle overload ability to determine 1-6 RM and train once a week. It is very important that the practice method should be correct, don't pull muscles excessively, and don't raise too much weight. Accidents are prone to happen when an error occurs.

We can rest 5-10 minutes between the same practices in each group, and take 3-4 training classes a week. If there is not enough time to recover between each group of training and training classes, severe isotonic training can make the person very tired, and it is easy to cause muscle pain. The reason is that the time of isotonic training is generally longer than isometric training, the energy consumption and the lactic acid produced is also more than isometric training, therefore, a long time is needed to recover and rest after a isotonic training. According to the purpose of each training classes, the isotonic training class should be in 1-2 hours or so, while the isometric training class just needs 30 minutes or less.

Big muscles should be trained firstly, because the force of isotonic training will be affected by the limitation of some weak muscles. Small muscles fatigue resistance ability of isotonic training will be higher than isometric training or isodynamic training. The trainings like bench press, squatting, crouch, push or inclined plate should be practiced before dumbbell, sit-ups, lift heel. That is to say, some large muscle groups' exercises, such as bench 
press, squatting, should be practiced firstly.

Normal training classes should be taken twice a week in order to maintain the growth of isotonic strength. If the times of training are less than twice a week, you will find that the growth strength will disappear in a month or so. On the other hand, it cannot increase the strength, only can maintain the strength in the early stages of the training for training twice a week.

\section{The General Principles of Isodynamic Training}

Isodynamic strength training has the advantages of both isotonic training and isometric training. In some ways, isodynamic training should be the biggest force (isometric training) within the scope of the whole action (isotonic training). The research of isodynamic training is not enough; we only introduce the general situation here. In order to increase strength faster, 4-5 isodynamic trainings can be taken every week, and the biggest dynamic contraction is 1-3 seconds. Action should be completed quickly for each of the biggest contraction in order to develop complex fast action. This view is based that on the whole range a maximum strength should be executed at every point, while doing these actions, the muscles' work time should not be too long to lead to muscle fatigue. The muscles in maximum static contraction will generally fatigue about in 10 seconds, three seconds is therefore more appropriate, while for some action one second is enough. For the complex movements, like baseball pitching, the trainers should be not only train the strength, but also train the fast control ability of the nervous system. It is ideal that both and can be practiced on the same equipment at the same time. Researchers believe that muscles can develop strength via fast contraction, and the effect of fast contraction is better than slow contraction.

The slower biggest contraction is close to isometric training, the time required is shorter than isotonic training. In each technical training, it is suggested that the frequency of isodynamic training should be more than isotonic training because the time is shorter. This opinion seems to be reasonable, because the recovery time is shorter between the isometric training classes. It is generally believed that the recovery time between the same actions should be 2-5 minutes. 1-2 regular training sessions per week is enough in order to keep the growth strength of isodynamic training. This means to keep a compromise of the isometric strength training and isotonic strength training.

\section{The Choice of Training Methods}

The above each training strength method has its advantages and disadvantages. No matter which method you use, the strength can be increased as long as the muscles are under overload training. The most important thing is to let those trainers choice their trusted training method and be voluntary to take several months to develop basic strength.

The advantages of isometric training include the following items. It is not necessary to spend much training time, and don't need to use expensive instrument equipment. The trainers can choose any area for training, and muscle ache is less after training. The muscle strength is easy to maintain. Its defects are: The trainers could not enhance strength within the whole scope of the action, unless it is reinforced in every point, but this will waste a lot of time. The training method has less effect on the nervous system. The training progress is difficult to be evaluated if there is no tensioner or test instrument of power. Some-times, isometric training can cause high blood pressure. Generally speaking, the growth strength of the isometric training is less than isotonic training or isodynamic training.

Isotonic training method is the most commonly used method for weightlifters to train strength. Isotonic training can develop muscle strength within the whole scope of the action, and has the same training effect on the nervous system as muscles. With the increasing of weight, muscle strength can be constantly and obviously improved. Isotonic training is more interesting than isometric training, and not easy to make people tired. Isotonic training method is also applicable to all sports, the trainers can use different instruments to practice (all kinds of ball, barbell, throwing equipment and pulley, etc.), but the isotonic training method is relatively expensive, because special equipment need to be manufactured according to the requirements of various actions. Sometimes muscle sore or injured may be caused after training because the choice of weight is too big. Weight lifting athletes have to run the risk of muscle injury, or the risk of pressure or touch injury by barbell, if they cannot lift the weight because a piece of muscle strength is weak doing action. We can spend 1-2 hours on one isotonic training session. The barbell or dumbbell's weight can be replaced during the process for continuous training. Isotonic training' effect on weaker muscles in the action scope is obvious; this is not a limit training for the whole range.

Isodynamic training is a new kind of training method and does not pass full of test like other methods. To a 
certain degree, isodynamic training combines the advantages of the isometric and isotonic training methods, while its disadvantages are less than the two methods. Isodynamic training can train muscles in different angles and make the biggest resistance, it takes less time than isotonic training. The actions can be completed in different speed in isodynamic training, and the muscles' damage and sore caused is less than isotonic training. The price of isodynamic training is close to the value of a good barbells and dumbbells. But its advantage is that we don't need to transform the weight of the instrument or move equipment when one kind of practice transfer to another or the trainer becomes a person from another person. Not only the strong leg muscles but also the small finger muscles can be practiced on the same instrument. The trainers can also read or record the specific number on the scale of training instrument to evaluate the efficacy of the training. In a word, the isodynamic training can cause the trainers to do their best to fight for the best effect.

Most of the isodynamic training instruments are very expensive. Therefore, it is more necessary to use them in the rehabilitation ward of the hospital than the general university or the family.

\section{The Effects of Age and Gender on Strength Training}

The volume of a muscle, the maturity of the nervous system and the influence of male hormones are closely related to gender and age. The strength growth of male around the age of 25 is $100 \%$, because in this age the strength growth by training is biggest (This does not mean that the muscle strength of this age is $100 \%$, it means that its biggest strength growth is $100 \%$, because our calculation method is that the test muscles whose strength from small to large is shown by the percentage of start strength).

It seems that the muscle training ability is also affected by the size of muscles between different sex and age, because the growth of the largest power is found in bigger muscles. To a certain extent, these muscles are usually under stress stimulation and regular training virtually in daily life, while the small muscles relatively lack of this kind of stress stimulation. Therefore, the strength growth percentage of thigh muscles is bigger than the finger muscles. The muscle strength of male is bigger that female, it is natural and strength training acceptability of male is bigger.

The same applies to the influence of the muscles' volume in all age groups on strength. Older people have more muscle, therefore, they has larger acceptability to the training, but training skills grow faster than muscle volume, probably order people around 30 years old is easier to accept the strength training because of the mature nervous system.

In conclusion, people with small strength in the beginning can get a larger growth percentage of strength after training, when the train reaches a certain level; it is difficult to increase strength. It is significant that the high levels weightlifters can maintain the growth strength several years.

Sports teachers and coaches, especially the women teachers and coaches, should be familiar with the differences of ability training acceptable between different age groups and gender. Once again, there are a lot of variability between different gender and age. Wise teachers and coaches should be good at discovering the individual differences, and try to find out the best way suitable for their personal strength growth correspondingly.

\section{References}

Chen, Y., \& Liu, J. G. (2008). Development of physical strength training for track athletes. 12th National Sports Biomechanics Academic Exchange Conference Papers Series.

Li, S. (2006). A new point of view for strength training scientifically. Journal of Guangzhou Physical Education Institute, (6).

Li, Z. H., \& Huang, G. Q. (2004). Discuss on the movement load control of modern strength training. 7th National Conference of Sports Science Abstracts, (2).

Song, S. G. (2002). The effects of strength training in upper limb muscle on javelin result. 10th national academic exchange conference proceedings of sports biomechanics.

Tan, T. C. (2011). Core strength training. Physical medicine and rehabilitation medicine in Zhejiang province in 2011 academic conference proceedings and rehabilitation progress in the new class.

Zhou, A. G., \& Zhao, C. S. (2007). The main problems existing in the strength training. 8th National Conference of Sports Science Abstracts, (2).

\section{Copyrights}

Copyright for this article is retained by the author(s), with first publication rights granted to the journal.

This is an open-access article distributed under the terms and conditions of the Creative Commons Attribution license (http://creativecommons.org/licenses/by/3.0/). 